\title{
Combatting Opioid Overdoses in Ohio: Emergency Department Physicians' Prescribing Patterns and Perceptions of Naloxone
}

\author{
Jonathan Penm, BPharm (Hons), PhD', Neil J. MacKinnon, BSc (Pharm), MSc (Pharm), PhD², \\ Michael S. Lyons, MD, MPH ${ }^{3}$, Erica Tolle, PharmD ${ }^{4,5}$, and Gregory T. Sneed, PharmD ${ }^{2}$ \\ ${ }^{1}$ Faculty of Pharmacy, The University of Sydney, Sydney, NSW, Australia; ${ }^{2}$ College of Pharmacy, University of Cincinnati, Cincinnati, OH, USA; \\ ${ }^{3}$ College of Medicine, University of Cincinnati, Cincinnati, OH, USA; Institute for Wellness and Education, Woodstock, GA, USA; ${ }^{5}$ Physician's \\ Pharmacy, Austell, GA, USA.
}

KEY WORDS: drug abuse; emergency medicine; practice variation; substance abuse; survey research.

J Gen Intern Med 33(5):608-9

DOI: $10.1007 / \mathrm{s} 11606-018-4353-6$

(c) Society of General Internal Medicine 2018

\section{INTRODUCTION}

Opioid-related drug overdoses account for over $60 \%$ of drug overdose deaths within the USA, representing a fourfold increase within the past 15 years. ${ }^{1}$ Ohio has the third highest opioid overdose death rate $(24.7$ deaths per 100,000$)$ in the country. ${ }^{2}$ The distribution of take-home naloxone to improve the chances of opioid overdose reversal has become an increasingly popular practice to combat this epidemic. The best predictor of future opioid overdose is a previous episode, for which patients are often seen by providers in the emergency department (ED). ${ }^{3}$ Yet, there is a lack of evidence evaluating the naloxone distribution practices of this group of healthcare professionals. The purpose of this study was to determine the prescribing patterns and perceptions of takehome naloxone for high-risk opioid overdose patients amongst ED physicians.

\section{METHODS}

A cross-sectional survey of ED medical directors or their designees was conducted to evaluate the implementation of Ohio opioid prescribing guidelines; the survey also examined take-home naloxone prescribing practices. The survey was distributed following Dillman's Tailored Design Method, augmented by telephone recruitment. ${ }^{4}$ All survey data was collected using REDCap (Research Electronic Data Capture) tools hosted at Cincinnati Children's Hospital Medical Center and analyzed using SE 13.1 (StatCorp, College Station, TX).

Semi-structured interviews with ED physicians and medical directors were conducted to gain an in-depth understanding of prescriber perception on the use of take-home naloxone for opioid overdose management. All interviews were conducted via telephone and audio recorded. Interviews

Published online February 28, 2018 lasted between 30 and $60 \mathrm{~min}$ and field notes were taken to augment interview data. All interview data was transcribed and analyzed in NVivo 10 (QSR International, Burlington, MA) using thematic analysis. ${ }^{5}$ This study was approved by the University of Cincinnati and Ohio Department of Health Institutional Review Boards.

\section{RESULTS}

Of the 163 EDs contacted to participate in the study, 134 responded to the naloxone-specific survey questions for an $82 \%$ response rate. In total, $57 \%$ of respondents were from urban hospitals and $43 \%$ were from rural hospitals. The survey results indicated that $57 \%$ of EDs had not provided a prescription for naloxone to patients at high risk of opioid overdose in the last month (Fig. 1), and 74\% had not provided take-home naloxone, either independently or as part of the state-funded naloxone distribution program (Project DAWN).

Thematic analysis of 20 individual interviews involving 8 ED physicians and 12 ED medical directors identified two main themes that contributed to the low frequency of naloxone distribution and prescription of take-home naloxone, including (1) prescribers' lack of education and awareness, and (2) prescribers' unconscious bias toward patients at high risk of opioid overdose. Overall, the majority of physicians supported naloxone availability in the community, but did not prioritize their own potential role in distribution (Table 1). However, some physicians expressed discomfort with the harmreduction aspects of take-home naloxone, indicating that it could increase willingness to engage in opioid abuse or that it would not affect long-term outcomes given that repeat overdose is common.

\section{DISCUSSION}

The idea that EDs frequently encounter patients at high risk for opioid overdose is both intuitive and demonstrated by available evidence. This report advances the literature base by quantifying the degree of ED participation in naloxone distribution and suggesting that cultural and operational 


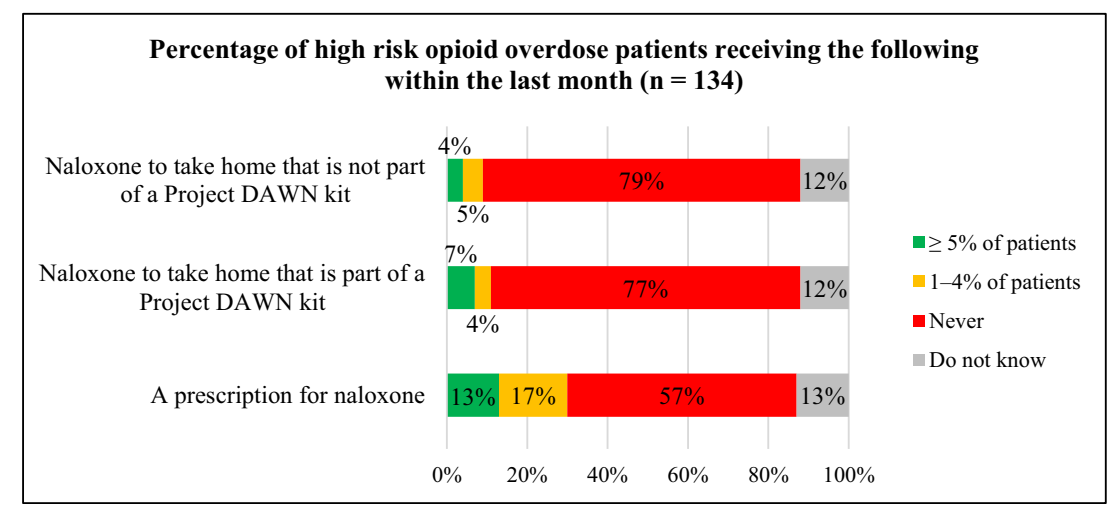

Figure 1 Naloxone practices for high-risk opioid overdose patients

interventions will be needed if implementation is to be accelerated. Our results are likely to be fairly generalizable, as the quantitative sample was quite broad and it is unlikely that EDs in regions with a lower prevalence of opioid overdose are more likely to have advanced their practice in naloxone distribution. Future work should not only develop interventions to promote naloxone distribution in EDs, but also determine the relative merits of naloxone prescriptions versus furnishing naloxone directly to patients prior to leaving the ED.

The majority of EDs do not currently provide take-home naloxone to patients at risk for opioid overdose. This is true even in Ohio, where opioid overdose is highly prevalent and statewide efforts, including funding and protocols to provide naloxone without a prescription from the treating provider, are in place to expand naloxone availability. Given the ability of bystanders to administer naloxone to reduce overdose mortality and ED access to at-risk populations, there is an urgent need to promote widespread expansion of ED participation in naloxone distribution efforts.

Table 1 Key informant interview themes

\begin{tabular}{ll}
\hline \hline Theme & Quotes \\
\hline Lack of education and & "PPrescribing take-home naloxone \\
awareness & is] not a bad thing necessarily. We \\
probably should be, but it's not part & of our practice." (Interview 15)
\end{tabular}

Unconscious bias toward patients at high risk of opioid overdose

1. Harm minimization will 1. "[Naloxone is] handed out to worsen outcomes

2. Patients will not benefit from take-home naloxone people who are at risk. I question whether or not it would lead to increased risky behavior if somebody knew that they had on-hand, the appropriate antidote." (Interview 18)

2. "I think the best reason to have naloxone is in case there's a pediatric case where a kid accidentally gets into narcotics... A lot of these people have terminal, I mean I hate to say it, but a lot of them have terminal opioid dependency." (Interview 7)
Contributors: We would like to acknowledge the American College of Emergency Physicians (Ohio Chapter) for their contributions to this work.

Corresponding Author: Gregory T. Sneed, PharmD; College of Pharmacy, University of Cincinnati, Cincinnati, OH, USA (e-mail: Gregory.Sneed@uc.edu).

Funding: This work received specific grant funding from the Ohio Department of Health. The funding source had no role in the design and conduct of this study; collection, management, analysis, and interpretation of the data; preparation, review, or approval of the manuscript; and decision to submit the manuscript for publication. This work was supported by the Grant or Cooperative Agreement Number, 6 NU17CE002738, funded by the Centers for Disease Control and Prevention. Its contents are solely the responsibility of the authors and do not necessarily represent the official views of the Centers for Disease Control and Prevention or the Department of Health and Human Services.

\section{Compliance with Ethical Standards:}

Presentations: This work was previously presented as a poster presentation at the American Society of Health-System Pharmacists Midyear Clinical Meeting and Exhibition in Orlando, Florida, in December 2017.

Conflict of Interest: Dr. Lyons has received investigator-initiated research funds paid to the institution from Brightview Health that were sourced in part from ADAPT Pharma, Inc. All remaining authors declare that they have no conflict of interest.

\section{REFERENCES}

1. Centers for Disease Control and Prevention. Wide-ranging online data for epidemiologic research (WONDER). Atlanta, GA: CDC, National Center for Health Statistics; 2016. Available at http://wonder.cdc.gov. Accessed 27 Nov 2017.

2. The Henry J. Kaiser Family Foundation. State Health Facts. Opioid Overdose Death Rates and All Drug Overdose Death Rates per 1000,000 Population (Age-Adjusted); 2015. Available at https://www.kff.org/other/ state-indicator/opioid-overdose-death-rates/. Accessed 27 Nov 2017.

3. Dwyer K, Walley AY, Langlois BK, et al. Opioid education and nasal naloxone rescue kits in the emergency department. Western Journal of Emergency Medicine. 2015;16(3):381.

4. Dillman DA, Smyth JD, Christian LM. Internet, Mail, and MixedMode Surveys, The Tailored Design Method. Hoboken, NJ: Wiley; 2014.

5. Braun V, Clarke V. Using thematic analysis in psychology. Qualitative research in psychology. 2006;3(2):77-101. 